\title{
Zur Theorie der elektrischen Nervenreizung durch Kondensatorentladung.
}

\author{
Von
}

\section{Arnold Eucken.}

Anlässlich einer zusammenfassenden Darstellung seiner Theorie der elektrischen Nervenreizung erwähnte Nernst kürzlich ${ }^{1}$ ), dass elektrische Reizversuche durch Kondensatorentladungen, wie sie bereits von zahlreichen Forschern angestellt wurden, zur Prüfung seiner Hypothese und der damit zusammenhängenden Fragen geeignet erschienen. Er wies gleichzeitig darauf hin, dass das Gesetz des elektrischen Schwellenreizes beim Kondensator voraussichtlich die Gestalt: $\nabla \sqrt{C}=$ Konst. annehmen würde. Auf seine Anregung hin habe ich inzwischen die Berechnung der Konzentrationsänderungen an Elektroden, die durch eine Kondensatorentladung polarisiert werden, durchgeführt ${ }^{3}$ ). Sie führt in der Tat zur obigen Annahme; für die maximale Konzentrationsänderung (Schwellenreiz) ergab sich nämlich ${ }^{4}$ ):

$$
c-c_{0}=0,541 \frac{v V \sqrt{C}}{\sqrt{k \pi} \bar{W}}=0,765 \cdot v \sqrt{\frac{E_{\text {Kond. }}}{k \pi W}},
$$

wobei $c-c_{0}$ die Konzentrationsänderung, $v$ das elektrochemischeÄquivalent, $\nabla$ die Spannung, $C$ die Kapazität des Kondensators, $k$ den Diffusionskoeffizienten des Depolarisators, $W$ den Widerstand des Stromkreises, $E_{\text {Kond. }}=\frac{V^{2} C}{2}$ die Energie des geladenen Kondensators bedeuten. Für den Zeitpunkt des Eintrittes der maximalen Konzentrationsänderung gilt die Beziehung:

$$
t=0,855 \mathrm{WC} \text {. }
$$

Für eine Gruppe von Stromstössen, bei denen sich die Stromstärke als Funktion eines mit $t$ verbundenen Parameters darstellen lässt $\left[J=J_{0} f(\mathrm{bt})\right]$, konnte allgemein nachgewiesen werden, dass
1) Pflüger's Arch. Bd, 122 S. 288.
2) A. a. O. S. 305 .
3) Eucken, Sitzungsber. d. kgl. preuss. Akad. d. Wissensch. 1908 \$. 524.
4) A. a. O. S. 529 Gl. 10. 
bei unveränderter Gestalt des Stromstosses die Konzentrationsänderung der durchgesandten Energie proportional ist ${ }^{1}$ ). Sobald man aber von einem Stromstoss zu einem solchen von anderer Gestalt übergeht, oder sobald sich der zeitliche Verlauf der Stromstärke nicht in der angegebenen einfachen Weise ausdrücken lässt, verliert dieser Satz seine Gültigkeit. Um z. B. ein und dieselbe Konzentrationsänderung hervorzurufen, bedarf es bei der Kondensatorentladung cet. par eines etwa 1,71 mal grösseren Energieaufwandes als bei einem konstanten Stromstoss, wie ein Vergleich der obigen Gleichung mit der für Gleichstrom geltenden, von $\mathrm{Nernst}^{2}$ ) abgeleiteten Beziehung:

zeigt.

$$
c-c_{0}=\nu J \sqrt{\frac{t}{\pi k}}=\nu \sqrt{\frac{\overline{E_{\text {Gizichstrom }}}}{k \pi W}}
$$

Zur Prüfung der aus der Nernst'schen Hypothese folgenden Forderung: $V \sqrt{C}$ müsse für den Schwellenreiz konstant sein, liegen bereits hinreichend Beobachtungen vor, von denen einige im folgenden wiedergegeben werden (Tab. 1-6).

Tabelle 1. Menschl. Nerv, Beob. Hoorweg ${ }^{3}$ ).

\begin{tabular}{c|c|c|c}
\hline$C\left(10^{-8} \mathrm{~F}.\right)$ & $V$ beob. & $V$ ber. & $V \sqrt{C}$ \\
\hline 20 & 9,0 & 9,0 & 40 \\
8 & 12,5 & 14,2 & 35 \\
5 & 16,0 & 18,0 & 36 \\
2 & 30,0 & 28,5 & 42,5 \\
1,5 & 36,0 & 33,0 & 44,0 \\
1 & 44,0 & 40,2 & 44,0 \\
& \multicolumn{2}{|c|}{$V=\frac{40,2}{\sqrt{C}}}$.
\end{tabular}

Tabelle 2. Froschnerv (ischadicus), Beob. Cybulski und Zanietowski ${ }^{4}$ ).

\begin{tabular}{l|l|l|l}
\hline$C\left(10^{-8} \mathrm{~F}.\right)$ & $V$ beob. & $V$ ber. & $\nabla \sqrt{C}$ \\
\hline 9,4 & 0,125 & 0,103 & 0,384 \\
2 & 0,195 & 0,224 & 0,276 \\
1 & 0,272 & 0,316 & 0,272 \\
0,5 & 0,468 & 0,447 & 0,331 \\
& \multicolumn{2}{|c}{$V=\frac{0,316}{\sqrt{C}}}$. \\
\end{tabular}

1) Eucken, a. a. 0. Gl. 7.

2) A. a. O. Gl. 18.

3) Pflüg er's Arch. Bd. 52 S. 87.

4) Pflüger's Arch. Bd. 56 S. 45. 
Tabelle 3. Menschl. Nerv, Beob. Zanietowski ${ }^{1}$.

\begin{tabular}{c|r|r|r}
\hline$C\left(10^{-8} \mathrm{~F}.\right)$ & $V$ beob. & $V$ ber. & $V \sqrt{C}$ \\
\hline 10 & 38 & 35 & 120 \\
5 & 45 & 49 & 101 \\
2 & 77 & 78 & 109 \\
1 & 110 & 110 & 110 \\
& \multicolumn{3}{|c}{$V=\frac{110}{\sqrt{C}}}$.
\end{tabular}

Tabelle 4. Menschl. Nerv (ulnarius), Beob. Waller ${ }^{2}$ ).

\begin{tabular}{c|c|c|c}
\hline \hline$C\left(10^{-8} \mathrm{~F}.\right)$ & $V$ beob. & $V$ ber. & $V \sqrt{\bar{C}}$ \\
\hline 80 & 10 & 8,7 & 89 \\
14 & 20 & 20,9 & 75 \\
5 & 30 & 35,0 & 68 \\
3,5 & 40 & 41,8 & 75. \\
1,5 & $70 \quad$ & 64,0 & 86 \\
& \multicolumn{2}{c}{$V=\frac{78,1}{\sqrt{0}}}$.
\end{tabular}

Tabelle 5. Froschnerv (gastrocnemius), Beob. Hermann ${ }^{3}$ )

\begin{tabular}{|c|c|c|c|}
\hline$C\left(10^{-8} \mathrm{~F}.\right)$ & $V$ beob. & $V$ ber. & $V \sqrt{C}$ \\
\hline $\begin{array}{c}20 \\
10 \\
5 \\
2 \\
1 \\
(0,1\end{array}$ & $\begin{array}{r}53,5 \\
67,6 \\
90,7 \\
139,5 \\
230,1 \\
1543,4\end{array}$ & $\begin{array}{r}48,4 \\
67,4 \\
96,5 \\
153,0 \\
216,0 \\
-\end{array}$ & $\begin{array}{l}239 \\
214 \\
203 \\
197 \\
230 \\
477,0)\end{array}$ \\
\hline
\end{tabular}

Tabelle 6. Froschnerv (gastrocnemius); Beob. Lapicque ${ }^{4}$ ). (Temp. $12^{\circ}$.),

\begin{tabular}{c|c|c|c}
\hline \hline$C\left(10^{-8}\right.$ F. $)$ & $V$ beob. & $V$ ber. & $V \sqrt{\bar{C}}$ \\
\hline 50 & 0,30 & 0,28 & 2,12 \\
10 & 0,55 & 0,59 & 1,74 \\
5 & 0,75 & 0,88 & 1,68 \\
2 & 1,38 & 1,39 & 1,95 \\
1 & 2,35 & 1,97 & 2,35 \\
& \multicolumn{2}{|c|}{$V=\frac{1,97}{\sqrt{C}}}$.
\end{tabular}

1) Zanietowski, Die Kondensatormethode. Leipzig 1906. S. 67.

2) Proc. of R. S. London vol. 65 p. 207.

3) Pflüger's Arch. Bd. 111 S. 537.

4) C. r. d. l. Société de Biologie t. 62 p. 37. 
Wie aus den Tabellen ersichtlich, zeigt die Grösse $V \sqrt{C}$ bei raschen und langsamen Entladungen (kleine und grosse Kapazität) höhere Werte als bei Entladungen von mittlerer Dauer; die durchgesandte Energie durchläuft demnach offenbar ein, wenn auch wenig ausgeprägtes Minimum, eine Erscheinung, der eine Reihe von Autoren eine hohe Bedeutung beimisst. Nach der Nernst'schen Hypothese ist anzunehmen, dass nur in der Näbe dieses wenig ausgeprägten Energieminimums der Nervenreiz unmittelbar von der durch den Stromstoss verursachten Konzentrationsänderung abhängt, dass aber bei langsamen und sehr raschen Entladungen Erscheinungen hinzutreten, die das Grundphänomen verdecken; diese sollen im folgenden besprochen werden.

Die Erscheinung erstens, die sich bei langsamen Entladungen geltend macht, bezeichnet Nernst als Akkommodation des Nervs. Ihre Haupteigenschaften, namentlich wie sie bei Versuchen mit Gleichstrom und Wechselstrom hervortreten, sind bereits von Nernst') beschrieben worden (z. B. Einfluss der Temperatur usw.).

Es möge daher nur eine bei den Kondensatorentladungen hervortretende Eigentümlichkeit der Akkommodation erwähnt werden, die aus einigen Beobachtungen Lapicques hervorgeht ${ }^{2}$ ). Dieser Forscher stellte fest, bis zu welchem Zeitpunkte die Kondensatorentladung physiologisch wirkt. Die Versuche wurden in folgender Weise angestellt: Zunächst wurde für eine bestimmte Kapazität die zum Schwellenreiz nötige Spannung gesucht. Dann wurde bei derselben Spannung und Kapazität nur ein Teil des Stromstosses von genau bekannter Dauer durch den Nerv geleitet; unterhalb einer bestimmten Zeit reagierte der Nerv nicht mehr (Tab. 7, dritte Reihe), oberhalb dieser Grenze verhielt er sich genau so, als ob der gesamte Stromstoss gewirkt hätte. In der vierten Reihe ist nach der Beziehung $t=0,855 W C$ die Zeit berechnet, die zur Erreichung der maximalen Konzentrationsänderung theoretisch nötig ist. Der Widerstand betrug $70000 \mathrm{Ohm}$.

Die Tabelle (S. 458) zeigt, dass der Nerv nicht auf die maximale Konzentrationsänderung anspricht; dieselbe wird so spät erreicht, dass sich der Nerv akkommodiert. Die Grösse der Konzentrationsänderung, auf die der Nerv tatsächlich reagiert, lässt sich nun ${ }^{3}$ ) unter Be-

1) Pflüger's Arch. Bd. 122 S. 311. 1908.

2) Journ. de Physiol. et de Pathol. gén. Nov. 1903, p. 1003.

3) Sitzungsber. S. 529 G1. 9. 
Tabelle 7. Froschnerv (gastrocnemius).

\begin{tabular}{|c|c|c|c|c|c|c|c|c|}
\hline \multirow{2}{*}{$C\left(10^{-8} \mathrm{~F}.\right)$} & \multirow{2}{*}{$V$} & \multirow{2}{*}{$\begin{array}{l}\text { Erforder- } \\
\text { liche Zeit } \\
\text { (sek. } 10^{-3} \text { ) }\end{array}$} & \multirow{2}{*}{$t \underset{\text { (ber.) }}{t(\max ) \cdot 10^{3}}$} & \multirow{2}{*}{$V \sqrt{C} \cdot 0,541$} & \multirow{2}{*}{$\overline{2} V \sqrt{C} \cdot \Phi\left(\frac{t}{W \bar{C}}\right)$} & \multicolumn{3}{|c|}{ Gleichstrom } \\
\hline & & & & & & $t \cdot 10^{3}$ & $\nabla$ & $V \sqrt{t}$ \\
\hline 100 & 0,106 & 1,8 & 60,0 & 0.586 & 0,168 & 1,85 & 0,1045 & 0,14 \\
\hline 10 & 0,123 & 1.54 & 6,0 & 0,211 & 0,158 & 1,55 & 0,109 . & 0,136 \\
\hline 5 & 0,140 & 1,35 & 3,0 & 0,168 & 0,150 & & & \\
\hline 2 & 0,185 & 0,93 & 1,20 & 0,142 & 0,136 & 0,98 & 0,130 & 0,125 \\
\hline 1 & 0,255 & 0,63 & 0,60 & 0,138 & 0,138 & 0,63 & 0,170 & 0,135 \\
\hline
\end{tabular}

nutzung der in der dritten Reihe angegebenen Zeiten berechnen. Das Resultat (dieser Konzentrationsänderung proportionale Werte) ist in der sechsten Reihe angegeben. Zwar gelingt es' auf diese Weise nicht, die Akkommodation vollständig zu eliminieren; immerhin wird wenigstens der Teil der Stromkurve ausgeschaltet, der den Nerv nach der Erregung, also physiologisch wirkungslos, durchläuft. So tritt denn auch hier das Grundgesetz (Konstanz der Erregungskonzentration) erheblich deutlicher zutage als in der fünften Reihe. Zum Vergleich ist in den letzten Reihen eine unter denselben Bedingungen gemachte Beobachtung mit Gleichstrom angeführt, aus der hervorgeht, dass die Akkommodation in beiden Fällen bei derselben Reizdauer beginnt ( $t=$ etwa $1 \cdot 5 \cdot 10^{-3}$ sek). Während beim konstanten Strom der gesamte Stromstoss zur Erzengung der Erregungskonzentration verwandt wird, geht, wie erwähnt, bei der Kondensatorentladung im Akkommodationsgebiet ein erheblicher Teil desselben unwirksam durch den Nerv. Daher wird in diesem Gebiet beim Kondensator eine sehr viel höhere Energie verbraucht als beim Gleichstrom. Das Energieminimum tritt folglich beim Kondensator schärfer hervor, und es hat den Anschein, als ob bei der Kondensatorentladung der Nerv gegenüber der Akkommodation empfindlicher sei als beim Gleichstrom.

Bei Beantwortung der zweiten Frage, wie die Abweichungen vom Grundgesetze bei raschen Entladungen zu erklären sind, sind zwei Möglichkeiten zu unterscheiden: dieselbe kann entweder auf einer 'physiologischen oder auf einer physikalischen Ursache beruhen.

Einerseits ist es denkbar, dass der Nerv nur dann auf den Reiz d. h. die Konzentrationsänderung anspricht, wenn dieselbe eine gewisse Zeit bindurch besteht. Namentlich bei Versuchen mit Wechselströmen könnte sich möglicherweise ein derartiger Einfluss der Zeit bemerkbar machen ${ }^{1}$ ), da die Konzentrationsänderungen in diesem Falle nur

1) Vgl. Nernst, Pflüger's Arch. a. a. O. S. 300. 
sehr kurze Zeit bestehen bleiben. Anders liegen die Verhältnisse bei einfachen Stromstössen, wie Kondensatorentladuug und Gleichstrom. Hier. wird das Maximum der Konzentrationsänderung in dem Augenblicke erreicht, wo der Stromstoss schon nahezu oder vollständig vorüber ist. Dann wird die Konzentrationsänderung sich wieder allmählich ausgleichen, und dieser Ausgleich ist (anders als bei Wechselstrom) unabhängig von dem ursprünglichen Stromstoss. Die Dauer des Ausgleiches hängt in erster Linie von der Grösse der (als elektrolytischer Trog betrachteten) Nervenzelle ab. Im allgemeinen wird anzunehmen sein, dass wenigstens bei einigermaassen kurzen Stromstössen die Erzeugung der Konzentrationsänderung weit rascher vor sich geht als ihr Abklingen. In diesem Falle würde also die maximale Konzentrationsänderung stets nahezu dieselbe Zeit bestehen und Gelegenheit haben, auf den Nerv zu wirken, gleichgültig, ob ihre Erzeugung mehr oder weniger rasch erfolgt ist.

Wahrscheinlicher als die Zurückführung jener Abweichung auf eine derartige physiologische Ursache erscheint eine physikalische Erklärung. Es handelt sich hierbei um die Frage: wird bei sehr kurzen Stromstössen bei konstant gehaltener Energie die Erregungskonzentration überhaupt erreicht, oder ist sie kleiner, als nach der Theorie zu erwarten ist? Wie erwähnt, hängt die Konzentrationsänderung in hohem Maasse von der Form der Stromkurve ab. Nun liegt die Annahme nahe, dass dieselbe bei kurzen Stromstössen deformiert wird. Bei raschen Kondensatorentladungen würde diese Deformation in erster Linie dem Einfiuss der Selbstinduktion [möglicherweise auch der Kapazität ${ }^{1}$ )] des Stromkreises zuzuschreiben sein. Zur Berechnung des Vorganges müsste man somit zu einer anderen Formel für die Stromkurve übergehen; z. B. Kondensatorentladung mit Selbstinduktion:

$$
J=\frac{\nabla e^{-\frac{W}{2 S}} t}{\sqrt{w^{2}-4 \frac{S}{C}}}\left(e^{+t \sqrt{\frac{W^{2}}{4 S^{2}}-\frac{1}{S C}}}-e^{-t \sqrt{\frac{W^{2}}{4 S^{2}}-\frac{1}{S C}}}\right)
$$

Da $t$ mit zwei verschiedenen Parametern verbunden ist, verliert der Satz, dass die Energie des Stromstosses zur Erzeugung der Erregungskonzentration konstant sein muss, seine Gültigkeit. Dass

1) Vgl. Zanietowski, a. a. 0. S. 16. 
eine derartige Deformation bei gleichem Energieverbrauch eine geringere Konzentrationsänderung zur Folge haben würde als eine nichtdeformierte Kurve, erscheint von vornherein einleuchtend. Denn je mehr die Stromkurve beim Durchgang der gleichen Elektrizitätsmenge ( $V$ und $C$ als konstant vorausgesetzt) verlängert wird (Einfluss der Selbstinduktion), desto geringer ist die durch sie bewirkte Konzentrationsänderung. Eine bestimmte Elektrizitätsmenge häuft nämlich eine bestimmte Menge des Elektrolyten an der Elektrode an; je länger aber diese Anhäufung dauert, desto mehr geht inzwischen durch Diffusion verloren. Beispiel: konstanter Stromstoss:

$$
c-c_{0}=\frac{\nu Q}{\sqrt{\pi k t}} ; Q=J t
$$

Dass allein die Selbstinduktion die Störungen bewirkt hat, muss als unwahrscheinlich bezeichnet werden, da man sonst dem Selbstinduktionskoeffizienten der Stromkreise der verschiedenen Beobachter unwahrscheinlich hohe Werte zuschreiben müsste ${ }^{1}$ ). Dass aber tatsächlich sehr ernebliche Deformationen der Stromkurve einzutreten pflegen, beweisen einige Versuche wiederum Lapicques ${ }^{2}$ ). Wie bei den Versuchen in Tab. 7 wurde nur ein Teil des Kondensators entladen. Aus der Zeitdauer des Stromschlusses, dem Widerstand usw. war die durchgesandte Elektrizitätsmenge zu berechnen; mit Hilfe eines ballistischen Galvanometers wurde dieselbe direkt gemessen. Es ergab sich eine regelmässige Abweichung; bei raschen Entladungen war die tatsächlich entladene (gemessene) Elektrizitätsmenge geringer als die nach der-logarithmischen Formel berechnete. Er selbst schliesst hieraus: „C'est-à-dire qu'il y a un retard à l'éta-

1) Nimmt man z. B. an, dass in Tabelle 5 bei den letzten eingeklammerten Werten die Stromkurve durch die Selbstinduktion bereits so weit deformiert gewesen sei, dass bei einer noch kleineren Kapazität eine oszillierende Entladung eingetreten wäre, dass also etwa für $C=1: 10^{-9} \mathrm{~F}$ der Ausdruck $\sqrt{\frac{W^{2}}{4 S^{2}}-\frac{1}{S C}}$ verschwinden würde, so müsste $\frac{4 S}{C}=W^{2}$, oder da $W=22100 \Omega$ betrug, $S=\frac{1 \cdot 10^{-9} \cdot 4,89 \cdot 10^{8}}{4}=0,12 \mathrm{H}$ en ry gewesen sein. Es ist nicht anzumehmen, dass der Selbstinduktionskoeffizient einen derartig hohen Wert besass; selbst ein zehnmal kleinerer Wert muss als. unwahrscheinlich bezeichnet werden.

2) Journ. de Physiol. et de Pathol. gén., a. a. 0. 
blissement du courant (self et capacité du circuit). Je n'ai jamais pu rendre ce retard négligeable." Sehr deutlich tritt diese Verzögerung auch bei einem anderen Versuche hervor, den Lapicque in der bei Tab. 7 angegebenen Weise mit einer etwas raseheren Entladung anstellte: es berechnet sich die Zeit des Eintrittes der maximalen Konzentrationsänderung $\left(C=2 \cdot 10^{-8}, W=13500\right) \mathrm{zu}$ $0,23 \cdot 10^{-3}$ Sek., während Lapicque als erforderliche Zeitdauer $t=$ $0,51 \cdot 10^{-3}$ Sek. findet. Da nun offenbar die Versuche Lapicques zu den genauesten auf diesem Gebiete gehören, scheint die Annahme gerechtfertigt, dass auch bei anderen Beobachtern ähnliche Deformationen der Stromkurve eingetreten sind.

Auch auf der Seite der kurzen Stromstösse zeigt sich bei der Kondensatorentladung eine stärkere Abweichung als beim konstanten Strom, wie ein Vergleich der Tabellen $1-6$ mit den von Nernst angeführten Tabellen ${ }^{1}$ ) lehrt. Fasst man nun die Deformation der Stromkurve als Ursache des seheinbaren Versagens des Grundgesetzes auf, so ist dieses Verhalten ohne weiteres erklärlich. Eine Stromkurve ist nämlich gegen eine Deformation (durch Selbstinduktion usw.) um so empfindlicher, je steiler sie ansteigt, je grösser $\frac{d J}{d t}$ ist. Offenbar ist dieses bei der Kondensatorentladung in weit höherem Maasse der Fall als beim konstanten Stromstoss.

Obgleich somit die Möglichkeit einer rein physiologischen Ursache für das Zustandekommen der Abweichungen bei raschen Entladungen nicht in Abrede gestellt werden kann, scheint es doch die Hauptaufgabe bei späteren Versuchen zu sein, die Form der Stromkurve auch bei sehr raschen Entladungen sorgfältig zu beachten, entweder indem man ihre Gestalt nach irgendeiner bekannten Methode direkt feststellt, oder indem man sämtliche in Frage kommenden Grössen (Selbstinduktion, Kapazität) genau ermittelt und in Rechnung setzt.

Zur weiteren experimentellen Prüfung der Nernst'schen Hypothese wäre es von Wichtigkeit, die physiologische Wirksamkeit der verschiedenartigen Stromstösse untereinander zu vergleichen. Oben wurde angegeben, dass zur Erzeugung der gleichen Konzentrationsänderung die Energie einer Kondensatorentladung 1,71 mal grösser sein muss als die eines konstanten Stromstosses. Statt 1,71 berechnet sich aus Tabelle 7 bei $t=0,93 \cdot 10^{-3}$ Sek. der immerhin naheliegende 
462 Arnold Eucken: Zur Theorie der elektr. Nervenreizung etc.

Wert $\frac{\boldsymbol{E}_{\text {Kondensator }}}{\boldsymbol{E}_{\text {Gileichstrom }}}=\frac{3,43}{2,24}=1,49$. Indessen reichen die bisherigen experimentellen Untersuchungen nicht aus, um für diesen Spezialfall ein endgültiges Urteil über die Übereinstimmung zwischen Theorie und Erfahrung zu gewinnen. Dieses wird von den Resultaten zukünftiger Beobachtungen abhängen. 